\title{
Migratory fishbones in the pharynx: a report of two cases
}

\author{
Shuyi $\mathrm{Xu}^{1}$, Xiaomin $\mathrm{Li}^{1}$, Yue Peng ${ }^{1}$, Jing Yang ${ }^{1}$, Qianxu Liu ${ }^{1}$, Jiefeng Guo ${ }^{1}$, and Zhijian \\ $\mathrm{Yu}^{1}$ \\ ${ }^{1}$ Zhuhai People's Hospital (Zhuhai hospital affiliated with Jinan University)
}

May 12, 2021

\begin{abstract}
Fishbones migrated into surrounding tissues is relatively unusual. We present two cases of patients' migratory fish bones into the retropharyngeal space and hypopharynx. All fishbones were removed without complications. Early diagnosis of migratory fishbone and therapeutic management are essential for optimal patient survival.
\end{abstract}

\section{Introduction}

Foreign bodies (e.g., fish bones) stuck in the pharynx are the most common emergency cases in otorhinolaryngology practice. Sharp and pointed fish bones may damage the mucosa. In a majority of cases, however, these can be easily removed. In rare cases, fish bones can migrate to the different parts of the body causing local infection1, large blood vessel rupture ${ }^{2}$, and gastrointestinal perforation ${ }^{3}$ if not promptly managed.

Here, we describe two cases of patients' migratory fish bones into the retropharyngeal space and hypopharynx. We discuss our experience in treating these cases using a specific approach for each patient.

\section{Case presentation}

Case 1: Migratory fishbone in the retropharyngeal space

A 62-year-old man presented with sudden onset pharyngeal pain after eating fish at a local hospital. Fiberoptic laryngoscopy showed no abnormalities. Computed tomography (CT) revealed a foreign body lying transversely at the right piriform fossa. Painless gastroscopy was performed to remove the foreign body, however, no foreign bodies were detected. During gastroscopy, he presented with laryngeal edema, which required tracheal intubation.

Four days after admission, he was referred to our hospital for management. Cervical CT showed a foreign body in the right retropharyngeal space between the cervical vertebrae 1 and 2 (Fig. 1A and 1B). Subsequently, he underwent surgical exploration of the retropharyngeal space under general anesthesia. During surgery, the foreign body could not be detected in the plane of the second cervical vertebra. An extended longitudinal incision was performed to find the fishbone in the surrounding tissues. Fortunately, a fishbone was found in the plane of the third cervical vertebra. A one-week follow-up revealed the absence of pain and foreign body sensation.

Case 2: Migratory fishbone in the hypopharynx

A 52-year-old man was admitted to our department for throat pain, foreign body sensation, dysphagia, and mild sialorrhea for 3 days after eating fish. Cervical CT confirmed the presence of a linear radiopaque structure in the left laryngopharynx (Fig. 2A and 2B). Flexible fiberoptic laryngoscopy did not identify a foreign body in the area but this revealed the absence of mucosal lesions (Fig. 2C). To further ascertain the presence of a foreign body in the hypopharynx, a gastroscopy was performed showing a fishbone in the 
left piriform fossa (Fig. 2D). The fishbone pierced from the left piriform fossa into the posterior pharyngeal wall. The object was extracted using forceps. The patient immediately felt comfortable.

\section{Discussion}

Here, we describe two cases of migratory fish bones in the pharynx that were not observable via flexible fiberoptic laryngoscopy. We used a specific treatment for each patient. Fortunately, all patients had favorable outcomes.

Patients suspected of having fish bone impaction usually undergo physical and laryngoscopic examinations. A fishbone at the base of the tongue or hypopharynx usually requires flexible fiberoptic laryngoscopy for observation. However, it is difficult to determine the precise localization of fish bones in cases with an endoscopically undetectable fishbone. Sharp fish bones are more prone to cause mucosal damage. Moreover, muscle contractions after entering the pharynx may contribute to migration ${ }^{4}$. Migratory fish bones are associated with an increased incidence of complications. Therefore, it is important to establish this diagnosis early and accurately. CT is considered the most sensitive (sensitivity $=100 \%$ ) modality for detecting ingested fish bones in soft tissues ${ }^{5}$. Therefore, $\mathrm{CT}$ is recommended when suspecting the migration of ingested foreign bodies, as demonstrated in the present cases.

The retropharyngeal space consists of loose soft tissue between the buccopharyngeal fascia and the prevertebral fascia. Owing to this structure, fish bones can migrate freely into the retropharyngeal space. This was consistent with our observations on the first case. During the operation, it is difficult to identify the location of the fishbone in the retropharyngeal space. We suggest the use of CT to approximate the position of the fishbone, followed by an extended longitudinal incision to identify its exact location in the surrounding tissues.

The incidence of foreign bodies in the hypopharynx is common. However, complete visualization of the foreign body is usually limited by the anatomically closed hypopharynx in its resting state. Modified Killian's method can be applied to remove an endoscopically invisible fish bone ${ }^{5}$. Unfortunately, the second patient had severe sore throat symptoms upon head flexion and rotation. In this case, we removed the fishbone through gastroscopy, which fully exposed the hypopharynx. This suggests that gastroscopy may be a good alternative for the visualization and removal of fish bones in such cases.

\section{Conclusion}

This paper highlights the possibility of fishbone migration into the surrounding tissues, especially in cases where it cannot be identified on routine inspection. Early diagnosis of migratory fishbone and therapeutic management are essential for optimal patient survival.

\section{Author's contributions}

Shuyi Xu contributed to the discussion and edited the manuscript. Xiaomin Li, Yue Peng, Jing Yang, Qianxu Liu, Jiefeng Guo participated in the data collection. Zhijian Yu wrote the manuscript. All authors read and approved the final manuscript.

\section{References}

1. Wu E, Huang L, Zhou Y, Zhu X. Migratory Fish Bone in the Thyroid Gland: Case Report and Literature Review. Case Rep Med2018;2018:7345723.

2. Jiang D, Lu Y, Zhang Y, Hu Z, Cheng H. Aortic penetration due to a fish bone: a case report. $J$ Cardiothorac Surg 2020;15:292.

3. Wu CW, Chiu YW. Unintentional fish bone ingestion causing perforation of small intestine. Intern Emerg Med 2020.

4. Ho NH, Chang FC, Wang YF. Clinical Approaches to Migrating Ingested Foreign Bodies in the Neck. Ear Nose Throat J 2020:584320051. 
5. Kikuchi D, Ikeda M, Murono S. Endoscopic removal of a fish bone foreign body in the hypopharynx with the modified Killian's method. Auris Nasus Larynx 2020.

\section{Figure legends}

Figure 1. The fishbone in the retropharyngeal space. Sagittal (A) and axial (B) computed tomography (CT) images showing linear calcifications in the retropharyngeal space (red arrow).

Figure 2. The fishbone in the hypopharynx. Sagittal (A) and axial (B) CT images showing linear calcifications in the hypopharynx (red arrow). (C) Flexible fiberoptic laryngoscopy showing the absence of any foreign body. (D) Gastroscopic view of a fishbone in the hypopharynx (yellow arrow).
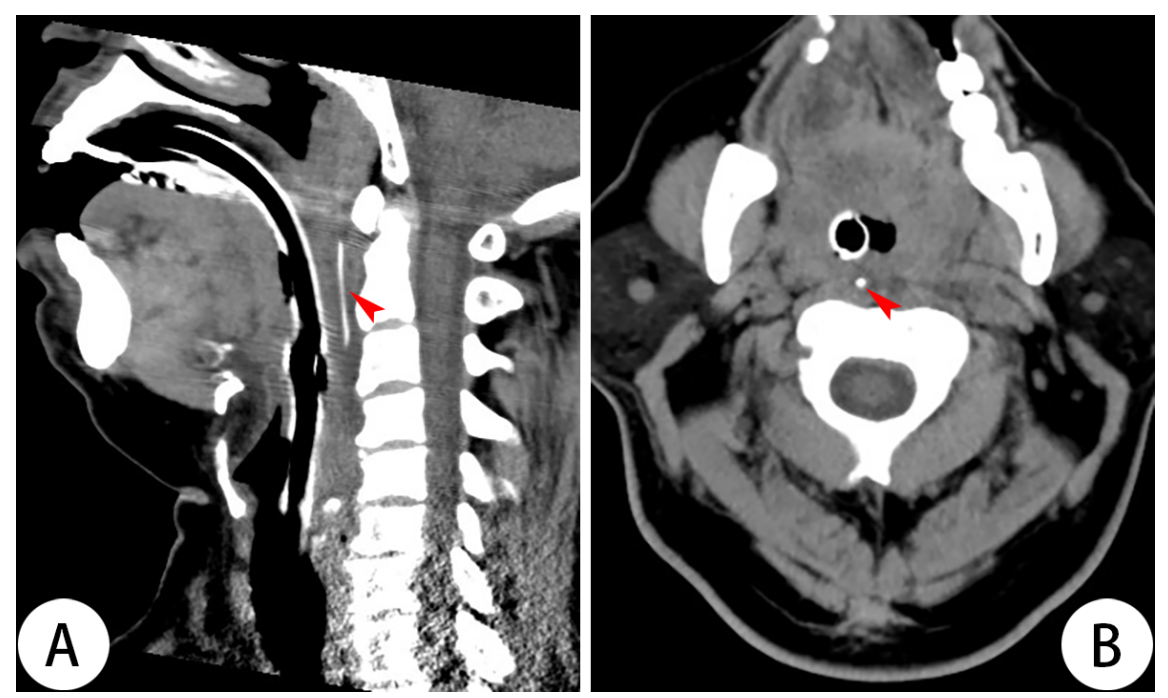


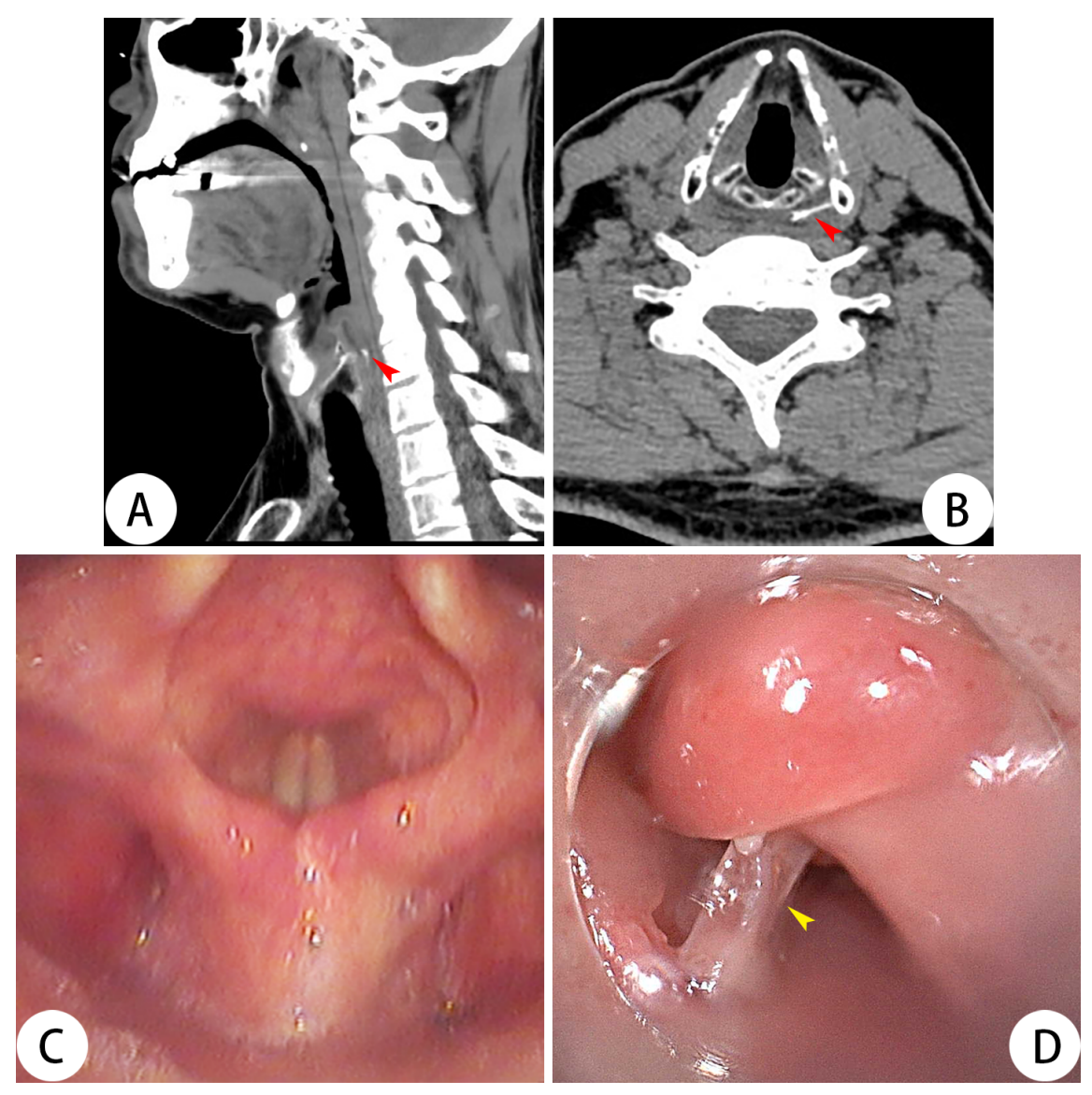

